\title{
Factors influencing the outcome of microsurgical transsphenoidal surgery for pituitary adenomas: A study on 184 patients
}

\author{
Kostas I. Lampropoulos, ${ }^{1}$ George Samonis, ${ }^{2}$ Panagiotis Nomikos ${ }^{1}$ \\ ${ }^{1}$ Department of Neurosurgery and Gamma Knife Radiosurgery, Hygeia Hospital, Marousi, Athens, ${ }^{2}$ Division of Medicine, \\ University of Crete, Heraklion, Crete, Greece
}

\begin{abstract}
OBJECTIVE: Evaluation of factors influencing the outcome of microsurgical transsphenoidal surgery in patients harbouring pituitary adenomas as well as of the efficacy and safety of this procedure. DESIGN: A total of 184 consecutive patients with pituitary adenomas, undergoing microsurgical transsphenoidal resection of their lesions from March 2004 to June 2011, were prospectively studied. Extent of tumour resection and disease remission were defined according to recently established radiological and hormonal consensus criteria. RESULTS: The study included 97 nonfunctioning and 87 functioning adenomas. A gross-total removal, as documented on postoperative imaging, was achieved in $67.4 \%$ of all patients. Residual tumour after surgery was detected in $37.1 \%$ of patients with nonfunctioning adenomas. The remission rates for patients with functioning adenomas, as documented by the last endocrinological evaluation, were $54.9 \%$ for growth hormone-secreting, $69.5 \%$ for adrenocorticotropin hormone-secreting, $\mathbf{7 2 . 7 \%}$ for prolactin-secreting and $\mathbf{1 0 0 \%}$ for thyroid-stimulating hormone-secreting, with two recurrences in patients with Cushing's disease. Multivariate analysis showed that factors influencing surgical outcome were cavernous sinus invasion, large tumour diameter $(\geq 25 \mathrm{~mm})$ and reoperation for the nonfunctioning adenomas, and cavernous sinus invasion as well as large tumour diameter $(\geq 25 \mathrm{~mm}$ ) for the functioning adenomas. In the latter cohort, predictors for endocrinological remission were maximum tumour diameter $(\leq 20 \mathrm{~mm})$ and reoperation. Postoperative complications were present in $3.3 \%$ of the cases. One patient developed epistaxis, two hemorrhage at the surgical field, one postoperative rhinorrhea, one postoperative permanent diabetes insipidus and one postoperative panhypopituitarism. CONCLUSIONS: Microsurgical transsphenoidal surgery is an effective and safe treatment in all patients with pituitary adenomas, except for prolactinomas responsive to medical therapy.
\end{abstract}

Key words: Outcome, Pituitary adenoma, Transsphenoidal microsurgery

Address for correspondence:

Kostas I. Lampropoulos, M.D., Department of Neurosurgery and Gamma Knife Radiosurgery, Hygeia Hospital, 4 Erythrou Stavrou Str., GR-15123 Marousi, Athens, Greece, Tel.: +30-210-6867107, e-mail: klampropoulos@hotmail.com

Received 21-06-2012, Accepted 07-01-2013 


\section{INTRODUCTION}

Pituitary adenomas, with an incidence of almost $25 \%$ in unselected autopsy series, ${ }^{1}$ account for 10 to $15 \%$ of all intracranial tumours ${ }^{2}$ and remain in many cases undiagnosed since they may not cause clinical symptoms. They are classified according to their secretory activity, with prolactinomas and null cell adenomas being the most frequent followed by growth hormone-, adrenocorticotropin hormone- and thyroid-stimulating hormone-secreting adenomas. Patients may present with well-defined clinical syndromes due to hormonal hypersecretion, including hyperprolactinemia, acromegaly, Cushing's disease and hyperthyroidism or with impaired pituitary function due to compression of the gland. Tumours with marked supra- and/or parasellar extension can cause severe headache, visual compromise and palsy of the oculomotor nerves.

Despite advances in the pharmacological and radiotherapeutic management, especially of hormonally active pituitary adenomas, ${ }^{3,4}$ surgery remains the treatment of choice for the majority of these tumours and the gold standard for hormonally inactive adenomas. ${ }^{5}$ The goal of surgical intervention is biochemical cure in addition to normal pituitary function preservation and decompression of the surrounding neural structures.

Surgical results, which are judged nowadays by strict endocrine criteria and sophisticated imaging, ${ }^{6,7}$ have endorsed transsphenoidal surgery as the most common and successful approach due to low risk of complications and because it can be applied in almost $90 \%$ of pituitary adenomas. ${ }^{5}$

The aim of this study was the evaluation and assessment of factors that influence the outcome of microsurgical transsphenoidal surgery (MTS) in a consecutive series of patients harbouring pituitary adenomas treated during the last seven years, using a standardized operative technique, and also the evaluation of the efficacy and the safety of the procedure.

\section{METHODOLOGY}

\section{Patients \& Methods}

Between March 2004 and June 2011, a total of 184 patients underwent endonasal transsphenoidal surgery for pituitary adenoma by the last author (PN) at the centre for pituitary surgery of Hygeia Hospital, Marousi, Greece. A standardized microsurgical approach was used, ${ }^{8}$ and in selected cases, with parasellar extension, the procedure was endoscope assisted. ${ }^{9}$ The main indication for the use of the combined microscopic and endoscopic approach was tumour extension outside the field of view of the microscope. This was particularly the case in tumours invading the parasellar area and those developed far laterally or anteriorly into the sphenoid sinus. Very large pituitary adenomas, requiring a transcranial approach, were excluded. These were mainly tumours demonstrating either a dumbbell shape with very narrow entrance of the sella, or tumours with larger suprasellar than intrasellar extension resulting in very small sella, or tumours with marked retrosellar portion.

\section{Preoperative Evaluation}

All patients underwent preoperative endocrinological, neuroradiological and neuro-opthalmological evaluation. Multiple measurements of plasma prolactin (PRL), growth hormone $(\mathrm{GH})$, insulin-like factor-I (IGF-1), GH level after oral glucose tolerance test (OGTT), corticotropin (ACTH), cortisol, 24-h urinary free cortisol (when Cushing's disease was suspected), thyrotropin (TSH), free thyroxine, luteinizing hormone $(\mathrm{LH})$, follicle-stimulating hormone (FSH), testosterone and estradiol levels were performed.

Additional preoperative data included age at operation, sex, symptoms at presentation, visual status and prior surgical procedures.

Magnetic resonance imaging (MRI) was performed on all but two patients (because of claustrophobia in the first and presence of pacemaker in the other) at the time of diagnosis. In these two patients, a highresolution computed tomographic (CT) scan of the hypothalamic-pituitary region was performed instead. Tumour size was classified according to its maximum diameter in two categories: microadenoma $(<10 \mathrm{~mm})$ and macroadenoma ( $\geq 10 \mathrm{~mm})$. For cavernous sinus invasion, the Knosp classification was used. ${ }^{10}$

The neuro-opthalmological workup included formal visual field testing and visual acuity assessment and was performed by a neuro-ophthalmologist. 


\section{Surgical Technique}

The original Cushing's method ${ }^{8}$ was used: the patient is positioned supine with the head tilted downwards about 10 degrees and the surgeon stands behind the patient. A unilateral paraseptal approach is employed. Depending on the nasal anatomy and the extent of the lesion, the mucosal incision is usually made in the vestibulum nasii along the cartilaginous nasal septum. A sublabial incision was applied in 10 cases of extended lesions. Under careful blunt dissection, the plane between cartilage and perichondrium is exposed. A mucosal tunnel is made without leaving this plane in order to prevent mucosal tearing. Resection of the anterior nasal spine of the maxilla gives better visualization of the tunnel. The basal cartilaginous septum is then mobilized and a Cushing-type speculum is inserted. Using the operating microscope, the tunnel is enlarged exposing the bony nasal septum, which has to be removed in order to reach the sphenoidal sinus. A speculum is introduced through one nasal cavity down to the anterior wall of the sphenoid sinus and the mucosa is coagulated and incised. ${ }^{8}$

The floor of the sphenoid sinus is then opened using a diamond drill and a larger self-retaining speculum is inserted. The sphenoid sinus is opened widely. The mucosa and all intrasphenoidal septae have to be removed, exposing the whole sella turcica from the sphenoidal plane to the clivus. In the case of incomplete pneumatization of the sphenoid sinus, the use of a drill is necessary in order to achieve such a wide exposure. The sellar floor is then opened and completely resected to the medial wall of the cavernous sinus. The basal dura is then opened and a small biopsy is taken to rule out tumour invasion histologically. The tumour is then removed using various curettes. During tumour removal, the diaphragm usually descends into the pituitary fossa. If this does not occur spontaneously, it can usually be accomplished by an increase in intracranial pressure using positive end-expiratory pressure (PEEP) ventilation or compression of the jugular veins. In the case of intraoperative cerebro-spinal fluid (CSF) leak, the surgical opening is sealed by two pieces of fascia lata fixed by fibrin glue. A lumbar catheter is then placed for CSF drainage until the third postoperative day. The mucosal incision is then closed and both nostrils are tamponaded for 24 hours. ${ }^{8}$ We applied
Medtronic ${ }^{\circledR}$ neuronavigation in 15 cases (especially in reoperations) and the procedure was endoscope assisted in 8 cases.

\section{Reconstruction of the Sellar Floor}

In 120 patients there was no need to use reconstruction material. The opening of the basal dura was covered only by a piece of an oxydized cellulose polymer (Surgicel ${ }^{\circledR}$ ) and covered by a hydrogel dural sealant $\left(\right.$ DuraSeal $\left.^{\circledR}\right)$. In 64 patients with intraoperative CSF-leak, reconstruction of the sellar floor by two pieces of the patient's fascia lata was necessary. The CSF-leak occurred during removal, with the most frontal part of the diaphragm being the most common site of leakage. Only in 7 patients was the leak due to extensive infiltration of the diaphragm by invasive growth of the lesions.

\section{Postoperative Evaluation}

The patient's postoperative neurological status, a full repeat endocrinological assessment, and postoperative MRI scans were reviewed. The criteria for disease control were tumour gross-total resection (GTR) in nonfunctioning adenomas and biochemical cure along with evidence of clinical remission in functioning adenomas.

In all cases, MRI scanning was routinely performed 3 months after surgery and was repeated at 1 year postoperatively, and then annually for the remainder of the followup period, unless there were indications for earlier imaging. GTR is defined as when the surgeon's intraoperative vision and postoperative imaging document no residual tumour. The resection is considered subtotal (STR) when more than $80 \%$ of the lesion has been removed and partial (PTR) when less than $80 \%$ has been removed. ${ }^{11}$

For patients with acromegaly, the normalization of the GH level ( $\mathrm{GH}<0.4 \mathrm{ng} / \mathrm{mL})$ and IGF-1 level (normal as adjusted for sex and age), as measured in the first 48 hours after surgery along with a GH nadir of $1 \mathrm{ng} / \mathrm{mL}$ or less after an OGTT performed at 6 weeks postoperatively, were considered as criteria for cure. ${ }^{12}$ For patients with Cushing's disease, an early morning cortisol level measurement $(<100 \mathrm{nmol} / \mathrm{L}$ requiring substitutive therapy) obtained in the first 48 hours after surgery, along with the suppression to low-dose dexamethasone and normalization of 
the 24-hour urinary free cortisol (both at 6 weeks postoperatively), were required. ${ }^{13}$ For patients with prolactinoma, PRL level after surgery $<20 \mathrm{ng} / \mathrm{mL}$ was considered an indicator of remission; ${ }^{14}$ and for patients with TSH-secreting adenomas, normalization of fT3, fT4 and TSH levels were required the day after surgery. All tests were repeated at least yearly or as required depending on the patient's clinical status.

All patients with preoperative visual deficits underwent formal neuro-ophthalmological evaluation postoperatively.

\section{Statistical Evaluation}

Continuous variables are displayed as mean values \pm standard deviation. Categorical values are shown as percentages. Tumour diameter between patients with favourable and unfavourable surgical and endocrinological outcome was assessed using a Mann-Whitney U-test. Multivariate logistic regression analysis was used to determine the effect of tumour invasion of the cavernous sinus, maximum tumour diameter, reoperation, suprasellar extension, sphenoid sinus invasion, and tumour apoplexy, on surgical outcome and endocrinological remission. A p value $<0.05$ was considered statistically significant. In the model used for this analysis, variables with $\mathrm{p}$ value $>0.1$ were considered not statistically significant and were not taken into account (removed from the model). Analysis was performed using commercially available software (MedCalc version 12.2.1.0).

\section{RESULTS}

\section{Patient Characteristics}

A total of 184 patients, with a mean age of 49.77 \pm 14.23 years, underwent resection of pituitary adenomas via the transsphenoidal route. There were 99 males. This series was composed of 97 patients with nonfunctioning adenomas, 51 with acromegaly, 23 with Cushing's disease, 11 with prolactinomas and 2 with TSH-secreting adenomas. Clinical presentation of patients with nonfunctioning adenomas was related to mass effect of the tumour (visual impairment and/ or hypopituitarism) in 73 of the 97, while the tumour was an incidental finding in 14 . The remaining 10 presented with tumour apoplexy, causing neurological symptoms. Most patients (92\%) with functioning tumours presented with hormone overproduction symptoms, whereas the remaining $(8 \%)$ with visual disturbances. In this series, 22 of the 184 patients had undergone a previous resection of their lesion, 13 of 97 with nonfunctioning and 9 of 87 with functioning adenomas. Mean duration of follow-up was $3.6 \pm 2$ years.

\section{Surgical Results}

\section{Nonfunctioning adenomas}

This cohort consisted of 97 patients with a mean age of $55.06 \pm 14.4$ years. There were 56 males. All patients harboured macroadenomas, with a median value of maximum tumour diameter on preoperative imaging of $25 \mathrm{~mm}$ (range 11-60). Invasion of the cavernous sinus was present in 33 of the 97 and extension to the sphenoid sinus in 19. According to the first postoperative neuroimaging study, GTR was demonstrable in 61 patients $(62.9 \%)$ and tumour remnants were seen in 36 (37.1\%). Mann-Whitney U-test revealed that patients with favourable surgical outcome had statistically significantly smaller adenomas compared to those with an unfavourable one (median value of maximum tumour diameter $24 \mathrm{~mm}$ vs $31 \mathrm{~mm}$, respectively; p <0.001) (Figure 1). Multivariate logistic regression analysis showed that tumour invasion of the cavernous sinus (OR 7.6; $95 \%$ CI 2.6-22.0; $<<0.001)$, relatively large tumour diameter $(\geq 25 \mathrm{~mm})$ (OR 0.2; 95\% CI $0.08-0.6$; $p$ $<0.05$ ), and reoperation (OR 6.72; 95\% CI $0.8-55.9$; $\mathrm{p}<0.05$ ), were associated with unfavourable surgical outcome, whereas suprasellar extension, sphenoid sinus invasion and tumour apoplexy had no statistically significant independent association with outcome.

Normalization of visual defects occurred in 21 of the 55 patients with visual disturbances, while improvement was observed in another 27. In 7, visual defects remained unchanged. They did not worsen in any patient. Third nerve palsies regressed after surgery in all 7 patients presenting this symptom.

Clinical and endocrinological evaluation revealed evidence of some degree of hypopituitarism preoperatively in 75 of the patients. Postoperative examination revealed impaired pituitary function 3 months after surgery in 53 and after a whole year in 50. At 12 months after surgery, anterior pituitary function was normalized in 14 , improved in 24 , remained unchanged 


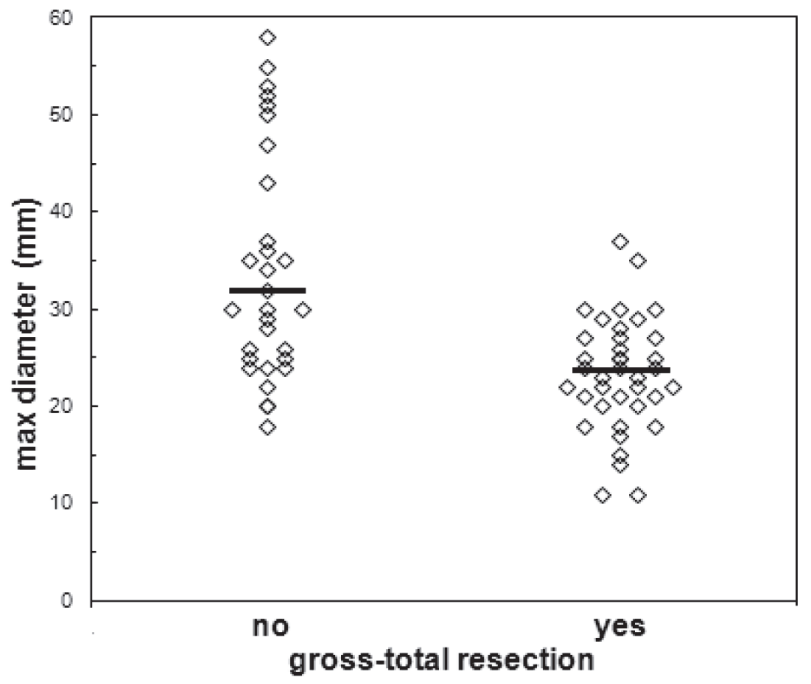

Figure 1. Association of tumour size with gross-total resection for nonfunctioning pituitary adenomas. Mann-Whitney U-test revealed that patients with favourable surgical outcome had statistically significantly smaller adenomas compared to those with an unfavourable one (median value of maximum tumour diameter $24 \mathrm{~mm}$ vs $31 \mathrm{~mm}$ (horizontal lines), respectively; p $<0.001)$.

in 36 and worsened in 1 of the 75 patients with some degree of preoperative hypopituitarism. One patient with an intra- and parasellar tumour histologically associated with a marked lymphocytic hypophysitis and normal preoperative pituitary function developed panhypopituitarism after surgery.

\section{Functioning Adenomas}

About two-thirds of patients with functioning tumours had macroadenomas. Invasion of the cavernous sinus was present in 20 of the 87 and suprasellar extension in 28. According to the first postoperative neuroimaging study, GTR was demonstrable in 63 patients $(72.4 \%)$. The rate of GTR was $88.9 \%$ for micro- and $65 \%$ for macroadenomas. Mann-Whitney U-test revealed that patients with favourable surgical outcome had statistically significantly smaller adenomas compared to those with an unfavourable one (median value of maximum tumour diameter $12 \mathrm{~mm}$ vs $25 \mathrm{~mm}$, respectively; $\mathrm{p}<0.001$ ) (Figure 2). Multivariate logistic regression analysis showed that tumour invasion of the cavernous sinus (OR 47.8; 95\% CI 5.4-421; p <0.001), as well as relatively large tumour diameter $(\geq 25 \mathrm{~mm})(\mathrm{p}<0.001)$, were associated with unfavourable surgical outcome, whereas

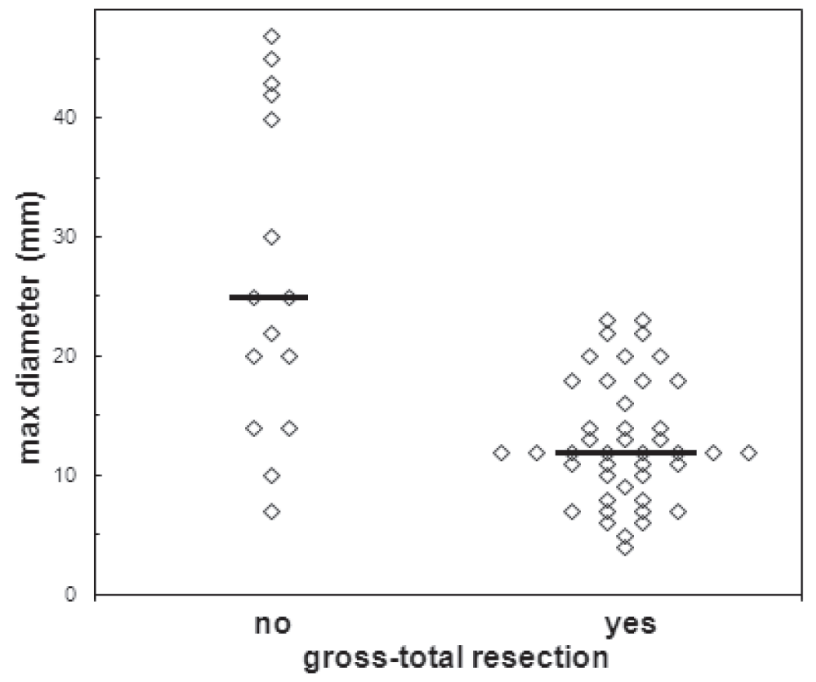

Figure 2. Association of tumour size with gross-total resection for functioning pituitary adenomas. Mann-Whitney U-test revealed that patients with favourable surgical outcome had statistically significantly smaller adenomas compared to those with an unfavourable one (median value of maximum tumour diameter $12 \mathrm{~mm}$ vs $25 \mathrm{~mm}$ (horizontal lines), respectively; $\mathrm{p}$ $<0.001)$.

suprasellar extension was not. Mann-Whitney U-test revealed that patients with endocrinological remission had statistically significantly smaller adenomas compared to those who were not cured (11 mm vs 15 $\mathrm{mm}$, respectively; $\mathrm{p}<0.05$ ) (Figure 3). Multivariate analysis showed that endocrinological remission was feasible for adenomas with smaller diameter $(\leq 20$ $\mathrm{mm})(\mathrm{p}<0.05)$ and reoperation was the only negative predictor for biochemical remission $(\mathrm{p}<0.05)$.

\section{Acromegaly}

This cohort consisted of 51 patients with a mean age of $44.61 \pm 10.68$ years. There were 32 males. Most patients presented with classic acromegalic symptoms and the median value of maximum tumour diameter on preoperative imaging was $13.5 \mathrm{~mm}$ (range 5-47). Out of the 51 patients, 41 had macroadenomas and in 17 the tumour had invaded the cavernous sinus. According to the first postoperative imaging, GTR was achieved in 70.6\% (36/51) and in 54.9\% (28/51) of the patients the endocrinological remission criteria were met. Biochemical cure was achieved in $80 \%$ of micro- and in $48.8 \%$ of macroadenomas. This latter result was due to the high incidence of tumour invasion of the cavernous sinus among patients of this category 


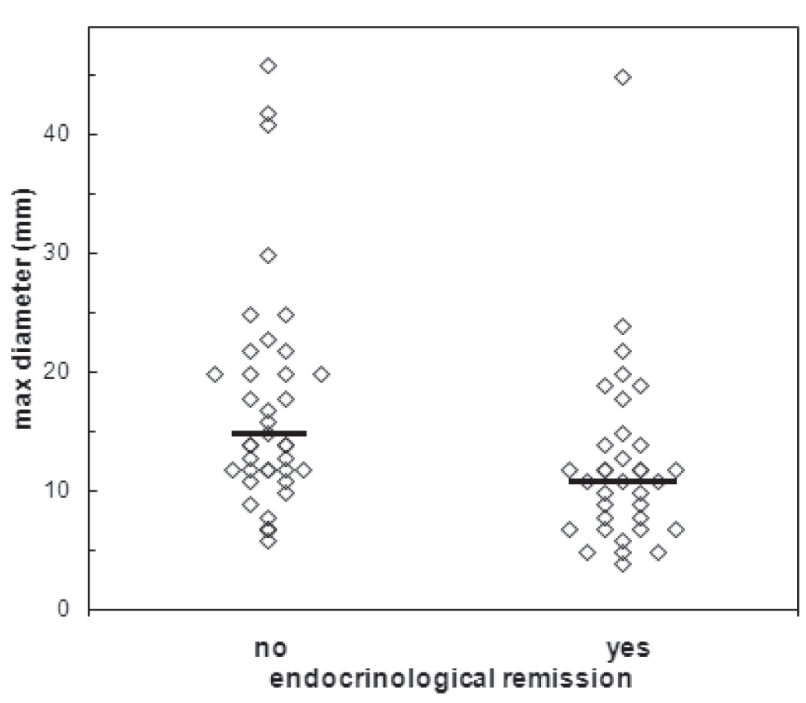

Figure 3. Association of tumour size with endocrinological remission for functioning pituitary adenomas. Mann-Whitney Utest revealed that patients with endocrinological remission had statistically significantly smaller adenomas compared to those who were not cured (median value of maximum tumour diameter $11 \mathrm{~mm}$ vs $15 \mathrm{~mm}$ (horizontal lines), respectively; $\mathrm{p}<0.05$ ).

(Table 1). All patients had normal pituitary function preoperatively, while one developed permanent diabetes insipidus post surgery. That patient required a second transsphenoidal operation in order to achieve biochemical remission.

Out of 23 patients who did not achieve endocrinological remission, 5 underwent supplementary therapy with Gamma Knife stereotactic radiosurgery.

\section{Cushing's Disease}

This cohort consisted of 23 patients with a mean age of $46.57 \pm 11.85$ years. Only 2 were males. All patients sought medical attention for classic cushingoid symptoms, except one who received the final diagnosis during the workup for low back pain. The median value of maximum tumour diameter on preoperative imaging was $9.5 \mathrm{~mm}$ (range 0-20). Only $39.1 \%$ of the lesions were classified as macroadenomas, while 5 had no pathological features on preoperative MRI. In these patients, the site of exploration of the pituitary gland was based on the results of the preoperative inferior sinus petrosus sampling. According to the first postoperative imaging, GTR was achieved in $73.9 \%(17 / 23)$, and in $69.5 \%(16 / 23)$ of the patients the endocrinological remission criteria were met. Biochemical cure was achieved in $77.8 \%$ of micro-, in $66.6 \%$ of macroadenomas, and in $60 \%$ of patients with no pathological features on preoperative imaging (Table 2). All patients had normal pituitary function preoperatively and none developed new endocrinological deficit post surgery. During the last followup 2 patients had developed recurrence (41 and 24 months after surgical treatment).

Out of 7 patients who did not achieve remission, 2 underwent supplementary therapy with Gamma Knife stereotactic radiosurgery.

Table 1. Acromegaly; remission rate in different categories of tumour following $\operatorname{MTS}^{1}(n=51)$

\begin{tabular}{|c|c|c|c|c|c|c|}
\hline \multirow[b]{2}{*}{ Tumour extension } & \multirow[b]{2}{*}{ Microadenomas } & \multicolumn{3}{|c|}{ Macroadenomas } & \multirow[b]{2}{*}{ Giant adenomas } & \multirow[b]{2}{*}{ Sum } \\
\hline & & is $^{2}$ & ps/sphe ${ }^{3}$ & $\mathrm{ss}^{4}$ & & \\
\hline Number of cases & 10 & 11 & 17 & 10 & 3 & 51 \\
\hline \multirow[t]{2}{*}{ Remission rate ${ }^{5}(\mathrm{n})$} & 8 & 7 & 8 & 5 & 0 & 28 \\
\hline & $(80 \%)$ & $(63.6 \%)$ & $(47 \%)$ & $(50 \%)$ & $(0 \%)$ & $(54.9 \%)$ \\
\hline
\end{tabular}

${ }^{1}$ Microsurgical Transsphenoidal Surgery, ${ }^{\text {intrasellar, }}{ }^{3}$ parasellar/sphenoidal, ${ }^{4}$ suprasellar, ${ }^{5}$ (basal GH $<0.4 \mathrm{ng} / \mathrm{ml}, \mathrm{OGTT}$ GH $<1 \mathrm{ng} /$ ml, normal IGF-I)

Table 2. Cushing's disease; remission rate in different categories of tumour following MTS ( $n=23$ )

\begin{tabular}{lcccc}
\hline Tumour extension & Microadenomas & Macroadenomas & No visible lesion & Sum \\
\hline Number of cases & 9 & 9 & 5 & 23 \\
Remission rate* $\left.^{*} \mathrm{n}\right)$ & 7 & 6 & 3 & 16 \\
& $(77.8 \%)$ & $(66.6 \%)$ & $(60 \%)$ & $(69.5 \%)$ \\
\hline
\end{tabular}

*cortisol $<100 \mathrm{nmol} / \mathrm{L}$, suppression to low-dose dexamethasone, normal 24-hour urinary free cortisol 


\section{Prolactinomas}

This cohort consisted of 11 patients with a mean age of $33.91 \pm 14.58$ years. Four were males. The majority $(81.8 \%)$ had received medical therapy to control excess prolactin secretion. Surgical treatment was decided because of insufficient control of hyperprolactinemia in 4 patients, enlargement of the adenoma despite medical treatment in 2 and intolerable adverse medication effects in 3 . Two patients underwent resection without previous medical treatment. The first presented with symptoms of tumour apoplexy (acute visual loss, complete pituitary insufficiency) while she was in the sixth month of gestation, and the second with rhinorrhea due to a giant invasive adenoma. The median value of maximum tumour diameter on preoperative imaging was 17 $\mathrm{mm}$ (range 7-44), while $81.8 \%$ of prolactinomas were classified as macroadenomas. According to the first postoperative imaging, GTR was achieved in $72.7 \%$ $(8 / 11)$, and in all these patients the endocrinological remission criteria were met. All patients, except one, had normal pituitary function preoperatively and none developed new endocrinological deficit post surgery. One patient with giant invasive adenoma developed postoperative rhinorrhea, which was surgically corrected.

Out of 3 patients who did not achieve remission, 1 underwent supplementary therapy with Gamma Knife stereotactic radiosurgery.

\section{Thyrotropin-secreting Adenomas}

Only 2 patients in this cohort underwent surgery, and according to the first postoperative imaging, a gross-total resection was achieved in both. The endocrinological results confirmed the normalization of TSH, fT3 and fT4 levels after surgery.

\section{Postoperative Complications}

No operative or perioperative death occurred in this series and there was no case of any kind of postoperative infection. Postoperative complications were present in $6(3.3 \%)$ patients. One developed epistaxis requiring embolism due to spheno-palatine artery pseudo-aneurysm, 2 hemorrhage at the surgical field treated with surgical evacuation, 1 postoperative rhinorrhea corrected surgically, 1 postoperative permanent diabetes insipidus and 1 postoperative panhypopituitarism. The two latter patients are receiving replacement therapy.

\section{DISCUSSION}

\section{Surgical Approach}

All patients treated underwent surgery using a microsurgical approach, which was assisted by endoscopy in a few cases with tumour extending far into the parasellar area and into the lateral or frontal part of the sphenoid sinus. To date, there is still lack of evidence that the endoscopic approach has been able to provide better results in the surgical treatment of pituitary adenomas in terms of normalization of hormonal oversecretion in functioning tumour or of more complete tumour removal in nonfunctioning adenomas as compared to microscopic surgery. ${ }^{9}$ The benefit of endoscopic visualization in transsphenoidal surgery is more prominent in the treatment of lesions other than adenomas, which extend far outside the confines of the sella. Pituitary adenomas usually develop in the intra- and suprasellar areas, which are perfectly in line of sight with the microscope. In the case of parasellar extension, usually associated with an invasive growth, visualization using the endoscope is clearly better. But even the use of the most sophisticated combined endoscopic and microsurgical methods has proven to be insufficient to remove the parasellar parts of the tumour in a way that no remnant is detectable in thin sliced postoperative MRIs and that in hormone-producing adenomas endocrinological cure can be achieved.

\section{Nonfunctioning Adenomas}

The aim of surgical treatment of nonfunctioning pituitary adenomas is the removal of as much of the adenoma as possible, leading to decompression of the adjacent nervous structures, along with preservation of normal pituitary function. MTS in the present series provided satisfactory control of tumour growth and resulted in clinical remission in a large percentage of the cases. The results in this study are comparable with those of other previously published series. ${ }^{15-20}$

Complete surgical removal of the adenoma was achieved in $62.9 \%$ of the patients in this study. The authors of two other large series reported similar results. ${ }^{16,19}$ These data suggest that successful removal of even large tumours can be accomplished in $>50 \%$ of cases in specialized centres. In the present series, all tumours were macroadenomas and the size of the treated lesions (median value of maximum tumour 
diameter $25 \mathrm{~mm}$ (range 11-60)) was larger than the sizes of the adenomas treated by other groups..$^{21,22}$

Impaired pituitary function can be restored after transsphenoidal surgery and this was the case of 14 $(18.7 \%)$ of the present patients who normalized and of the $24(32 \%)$ who had improved pituitary function, among the 75 patients with some degree of preoperative hypopituitarism. Similar results were reported by some authors, ${ }^{18-19}$ although in other series no functional improvement was observed. ${ }^{23}$ Permanent diabetes insipidus occurred in only one of the present patients, a consequence also reported in another series. ${ }^{19}$

Improvement of visual function occurred in $87 \%$ of the patients with preoperative disturbances and was similar to the improvement reported by other groups. ${ }^{17,22,24}$

Outcome was negatively influenced by three clinical factors, namely, cavernous sinus invasion, large tumour diameter $(\geq 25 \mathrm{~mm})$ and reoperation, as shown by multivariate analysis. Two other studies ${ }^{16,25}$ have also demonstrated that cavernous sinus invasion was the main negative predictor regarding complete removal. Patients who presented with pituitary apoplexy had a favourable outcome, with complete tumour removal. The small number of these patients cannot lead to a definite conclusion. However, we agree with the hypothesis of Losa and coworkers, ${ }^{16}$ that the features of apoplexy (massive necrotic and hemorrhagic changes of the tumour) may facilitate complete removal.

In the present series, reoperation negatively influenced outcome. No similar findings have been previously reported, but it is not clear whether this factor has ever been investigated in this context. It is likely that our department, representing a centre of pituitary surgery for the whole country, deals with a diversity of cases and, hence, many of them were operated on for the second or even third time. From a technical point of view, total removal in these cases is not feasible due to either difficulties in surgical exposure of the area, because of disturbed anatomy, or extensive bleeding, usually attributed to adhesions and previous use of materials for reconstruction of the sella during the first operation, such as glues and bone grafts.
In some studies, ${ }^{26-28}$ postoperative radiotherapy was performed in patients with residual tumours, taking into consideration that radiotherapy is effective in stabilizing tumour growth or inducing regression of the adenoma. The application of radiosurgery in cases of residual tumour leads to tumour control in more than $90 \%$ of all patients..$^{29-31}$ In the present series, postoperative radiation treatment was performed in 15 patients with residual tumour (10 received stereotactic radiosurgery with Gamma Knife and 5 conformal fractionated radiotherapy).

\section{Functioning Adenomas}

In the present cohort, endocrinological outcome was assessed according to the recently updated criteria for biochemical cure based on the latest consensus criteria of remission for acromegaly, Cushing's disease, and prolactinomas. ${ }^{12-14}$ In the recent past, there has been little universal consensus, making comparisons of outcome between different transsphenoidal series difficult.

\section{Acromegaly}

Despite the recent developments in medical therapy by the introduction of new agents and in radiotherapy by the use of stereotactically targeted therapy, transsphenoidal surgery remains the first-line treatment in all patients with acromegaly harbouring microadenomas, noninvasive macroadenomas, and tumours causing compression symptoms. ${ }^{6}$ Even in larger and invasive tumours unlikely to be controlled by surgery alone, debulking increases the proportion of patients in whom postoperative medical treatment results in normal IGF-1 levels, especially when more than $75 \%$ of the tumour is removed. ${ }^{32,33}$

MTS provides biochemical control in 33-95\% of patients. ${ }^{34-38}$ The best results are achieved in microadenomas and intrasellar macroadenomas (75-95\%). Remission rate tends to drop in more extensive adenomas, reaching $44.5 \%$ for suprasellar tumours in patients without, $33 \%$ in those with visual compromise, and $41.5 \%$ for tumours with parasellar and/or sphenoidal expansion. ${ }^{35}$

In the present study, biochemical cure was achieved in $54.9 \%$ of patients. The rate of endocrinological remission was $80 \%$ for patients harbouring microadenomas and was comparable with that reported 
by Nomikos and coworkers. ${ }^{35}$ In the present series, a large proportion of macroadenomas invading the cavernous sinus $(33.3 \%)$ ) were operated on and, hence, lower cure rates in $\mathrm{GH}$-secreting macroadenomas are reported as compared to the study by Abosch et al. ${ }^{39}$

In a large number of acromegalic patients there was a significant discrepancy between the results of postoperative MRI and the endocrinological remission. Despite the high rate of radiologically complete tumour removal (70.6\%), biochemical cure was documented in only $54.9 \%$. At present, MRI is thought to be the best imaging technique for evaluation of pituitary adenomas. ${ }^{40,41} \mathrm{MRI}$ is also recommended for followup of surgically treated patients. ${ }^{11}$ Residual or recurrent tissue of pituitary tumours is usually visible on postoperative MRI. ${ }^{42}$ However, postsurgical MRI lacks specificity. Postoperative changes may represent only scar tissue or material used for the reconstruction of the sellar floor and not residual adenomas. ${ }^{43-44}$ Local invasion of the walls of the cavernous sinus or of the osseous sellar floor is not always detectable on followup studies. Often, they can be detected in serial MRI scans. ${ }^{45,46}$ Therefore, the outcome of surgery regarding the control of the disease should only be based on biochemical results using the most current criteria for remission.

\section{Cushing's Disease}

MTS is the mainstay of treatment for Cushing's disease and can lead to an immediate normalization of cortisol levels along with improvement in symptoms. Remission rates after surgery vary significantly among studies, ranging from 42 to $95 \%$, with most between 70 and $85 \%$. . $^{47-52}$

In this study, biochemical cure was achieved in $69.5 \%$ of patients, with lower cure rate $(60 \%)$ in adenomas that were not visible on preoperative imaging. Endocrinological remission is known to be negatively affected by the poor preoperative visualization of the tumour. ${ }^{52}$ One of the two patients who were diagnosed with recurrence at followup had no identifiable lesion in the preoperative imaging.

\section{Prolactinomas}

To date, medical treatment is the favoured primary therapy for prolactinomas. Main indications of surgery are intolerance of medication and tumours not responding to dopamine agonists. Remission rates in large series of surgically treated prolactinomas vary between $54 \%$ and $86 \% .{ }^{53-56}$ In this study, biochemical cure was achieved in $72.7 \%$ of patients.

\section{Thyrotropin-secreting Adenomas}

Because of the rarity of such adenomas, our experience is limited to just two cases, and although treated with success, we cannot draw meaningful conclusions.

\section{Complication Analysis}

The experience of a well-trained surgeon is paramount in avoiding loss of time and major complications related to the approach. ${ }^{57}$ The rate of complications in the present study was comparable to previous large series. ${ }^{5}$ We had one patient $(0.5 \%)$ with delayed epistaxis, despite the meticulous cauterization of the posterior nasal branch of the spheno-palatine artery bilaterally. That patient required embolism due to arterial pseudo-aneurysm. CSF leak and meningitis are the most frequent complications in transsphenoidal surgery. ${ }^{57}$ Our low percentage $(0.5 \%)$ of postoperative rhinorrhea is explained not only by a careful surgical technique, but also by the placement of CSF lumbar external diversion after the end of the procedure, when intraoperative leak was present. Infectious complications were absent in the present series, despite the absence of preoperative prophylaxis with antimicrobial agents. The hemorrhagic complication $(1 \%)$ was as low as the most successful microsurgical series ${ }^{5}$ and the rates of permanent diabetes insipidus $(0.5 \%)$ and new anterior pituitary insufficiency $(0.5 \%)$ seem to be lower than in previous reports. ${ }^{5,57}$

In conclusion, the results of this study confirm the value, efficacy and safety of microsurgical transsphenoidal surgery for pituitary adenomas. The technique can achieve favourable removal and remission rates with very low morbidity. However, it should ideally be performed by an experienced pituitary surgeon. Taking into account the present results and those reported in the literature, microsurgical transsphenoidal surgery can be considered the first-choice therapy in all patients with pituitary adenoma, except for prolactinomas responsive to dopamine agonists.

\section{ACKNOWLEDGEMENTS}

The authors wish to thank their endocrinology colleagues, who referred their patients for surgical 
treatment and provided the requested follow-up data. The senior author (PN) would also like to express his appreciation and thanks to Professor Rudolf Fahlbush, his exceptional teacher and master of the management of patients with pituitary tumours.

\section{REFERENCES}

1. Burrow GN, Wortzman G, Rewcastle NB, Holgate RC, Kovacs K, 1981 Microadenomas of the pituitary and abnormal sellar tomograms in an unselected autopsy series. N Engl J Med 304: 156-158.

2. Asa SL, 1989 Tumors of the pituitary gland. In: third series (ed) Atlas of tumor pathology, Armed Forces Institute of Pathology, Washington DC: Fascicle 22.

3. Molitch ME, 1999 Medical treatment of prolactinomas. Endocrinol Metab Clin North Am 28: 143-169.

4. Losa M, Picozzi P, Motta M, Valle M, Franzin A, Mortini $\mathrm{P}, 2011$ The role of radiation therapy in the management of non-functioning pituitary adenomas. J Endocrinol Invest: 34: 623-629.

5. Mortini P, Losa M, Barzaghi R, Boari N, Giovanalli M, 2005 Results of transsphenoidal surgery in a large series of patients with pituitary adenoma. Neurosurgery 56: 1222-1233.

6. Melmed S, Colao A, Barkan A, et al, 2009 Guidelines for acromegaly management: an update. J Clin Endocrinol Metab 94: 1509-1517.

7. Nimsky C, von Keller B, Ganslandt O, Fahlbush R, 2006 Intraoperative high-field magnetic resonance imaging in transsphenoidal surgery of hormonally inactive pituitary macroadenomas. Neurosurgery 59: 105-114.

8. Buchfelder M, Fahlbusch R, 2002 The classic transsphenoidal approach for resection of pituitary tumors. Operat Techn Neurosurg 5: 210-217.

9. Dehdashti AR, Ganna A, Karabatsou K, Gentili F, 2008 Pure endoscopic endonasal approach for pituitary adenomas: early surgical results in 200 patients and comparison with previous microsurgical series. Neurosurgery 62 : 1006-1015.

10. Knosp E, Steiner E, Kitz K, Matula C, 1993 Pituitary adenomas with invasion of the cavernous sinus space: Magnetic resonance imaging classification compared with surgical findings. Neurosurgery 33: 610-617.

11. Lissett CA, Shalet SM, 2000 Management of pituitary tumours; strategy for investigation and follow-up. Horm Res 53: 65-70.

12. Giustina A, Chanson P, Bronstein MD, et al, 2010 A consensus on criteria for cure of acromegaly. J Clin Endocrinol Metab 95: 3141-3148.

13. Arnaldi G, Angeli A, Atkinson AB, et al, 2003 Diagnosis and complications of Cushing's syndrome: a consensus statement. J Clin Endocrinol Metab 88: 5593-5602.

14. Casanueva FF, Molitch ME, Schlechte JA, et al, 2006 Guidelines of the Pituitary Society for the diagnosis and management of prolactinomas. Clin Endocrinol (Oxf) 65: 265-273.

15. Shou XF, Li SQ, Wang YF, Zhao Y, Jia PF, Zhou LF, 2005 Treatment of pituitary adenomas with a transsphenoidal approach. Neurosurgery 56: 249-256.

16. Losa M, Mortini P, Barzaghi R, et al, 2008 Early results of surgery in patients with nonfunctioning pituitary adenoma and analysis of the risk of tumor recurrence. J Neurosurg 108: 525-532.

17. Ebersold MJ, Quast LM, Laws ER Jr, Scheithauer B, Randall RV, 1986 Long-term results in transsphenoidal removal of nonfunctioning pituitary adenomas. J Neurosurg 64: 713-719.

18. Marazuela M, Astigarraga B, Vicente A, et al, 1994 Recovery of visual and endocrine function following transsphenoidal surgery of large nonfunctioning pituitary adenomas. J Endocrinol Invest 17: 703-707.

19. Nomikos P, Ladar C, Fahlbusch R, Buchfelder M, 2004 Impact of primary surgery on pituitary function in patients with non-functioning pituitary adenomas - a study on 721 patients. Acta Neurochir (Wien) 146: 27-35.

20. Wilson CB, Dempsey LC, 1978 Transsphenoidal microsurgical removal of 250 pituitary adenomas. J Neurosurg 48: 13-22.

21. Jain AK, Gupta AK, Pathak A, Bhansali A, Bapuraj JR, 2008 Endonasal transsphenoidal pituitary surgery: is tumor volume a key factor in determining outcome? Am J Otolaryngol 29: 48-50.

22. Zhang X, Fei Z, Zhang J, et al, 1999 Management of nonfunctioning pituitary adenomas with suprasellar extensions by transsphenoidal microsurgery. Surg Neurol 52: 380-385.

23. Wichers-Rother M, Hoven S, Kristof RA, Bliesener N, Stoffel-Wagner B, 2004 Non-functioning pituitary adenomas: endocrinological and clinical outcome after transsphenoidal and transcranial surgery. Exp Clin Endocrinol Diabetes 112: 323-327.

24. Harris PE, Afshar F, Coates P, et al, 1989 The effects of transsphenoidal surgery on endocrine function and visual fields in patients with functionless pituitary tumours. Q J Med 265: 417-427.

25. Greenman Y, Ouaknine G, Veshchev I, Reider-Groswasser II, Segev Y, Stern N, 2003 Postoperative surveillance of clinically non-functioning pituitary macroadenomas: markers of tumor quiescence and regrowth. Clin Endocrinol (Oxf) 58: 763-769.

26. Chang EF, Zada G, Kim S, et al, 2008 Long-term recurrence and mortality after surgery and adjuvant radiotherapy for non-functional pituitary adenomas. $\mathrm{J}$ Neurosurg 108: 736-745.

27. Losa M, Mortini P, Barzaghi R, Franzin A, Giovanelli M, 2001 Endocrine inactive and gonadotroph adenomas: diagnosis and management. J Neurooncol 54: 167-177.

28. Park P, Chandler WF, Barkan AL, 2004 The role of radiation therapy after surgical resection of nonfunctional pituitary macroadenomas. Neurosurgery 55: 100-106. 
29. Pollock BE, Cochran J, Natt N, et al, $2008 \gamma$-Knife radiosurgery for patients with nonfunctioning pituitary adenomas: results from a 15-year experience. Int J Radiat Oncol Biol Phys 70: 1325-1329.

30. Sheehan JP, Kondziolka D, Flickinger J, Lunsford LD, 2002 Radiosurgery for residual or recurrent nonfunctioning pituitary adenoma. J Neurosurg 97: 408-414.

31. Sheehan JP, Niranjan A, Sheehan JM, et al, 2005 Stereotactic radiosurgery for pituitary adenomas: an intermediate review of its safety, efficacy, and role in the neu- rosurgical treatment armamentarium. J Neurosurg 102: 678-691.

32. Petrossians P, Borges-Martins L, Espinoza C, et al, 2005 Gross total resection or debulking of pituitary adenomas improves hormonal control of acromegaly by somatostatin analogs. Eur J Endocrinol 152: 61-66.

33. Colao A, Attanasio R, Pivonello R, et al, 2006 Partial surgical removal of growth hormone-secreting pituitary tumors enhances the response to somatostatin analogs in acromegaly. J Clin Endocrinol Metab 91: 85-92.

34. Abe T, Ludecke DK, 2001 Effects of preoperative octreotide treatment on different subtypes of $90 \mathrm{GH}$-secreting pituitary adenomas and outcome in one surgical centre. Eur J Endocrinol 145: 137-145.

35. Nomikos P, Buchfeder M, Fahlbusch R, 2005 The outcome of surgery in 668 patients with acromegaly using current criteria of biochemical cure. Eur J Endocrinol 152(3): 379-387.

36. Kaltsas GA, Isidori AM, Florakis D, et al, 2001 Predictors of the outcome of surgical treatment in acromegaly and the value of the mean growth hormone day curve in assessing postoperative disease activity. J Clin Endocrinol Metab 86: 1645-1652.

37. Shimon I, Cohen ZR, Ram Z, Hadani M, 2001 Transsphenoidal surgery for acromegaly: endocrinological follow-up of 98 patients. Neurosurgery 48: 1239-1243.

38. Beauregard C, Truong U, Hardy J, Serri O, 2003 Longterm outcome and mortality after transsphenoidal adenomectomy for acromegaly. Clin Endocrinol (Oxf) 58: 86-91.

39. Abosch A, Tyrrell JB, Lamborn KR, Hannegan LT, Applebury CB, Wilson CB, 1998 Transsphenoidal microsurgery for growth hormone-secreting pituitary adenomas: initial outcome and longterm results. J Clin Endocrinol Metab 83: 3411-3418.

40. Gao R, Isoda H, Tanaka T, et al, 2001 Dynamic gadolinium-enhanced MR imaging of pituitary adenomas: usefulness of sequential sagittal and coronal plane images. Eur J Radiol 39: 139-146.

41. Markus JB, Franchetto AA, Goh R, 1999 The use of intravenous contrast in postoperative MR imaging of pituitary macroadenomas. Clin Immunol 23: 5-10.

42. Kremer P, Forsting M, Hamer J, Sartor K, 1996 MR imaging of residual tumor tissue after transsphemoidal surgery of hormone-inactive pituitary macroadenomas; a prospective study. Acta Neurochir 65: 27-30.

43. Candrina R, Gasparotti R, Galli G, Rukgerri G, Bollati A, 1991 Comparison between computed tomography and magnetic resonance imaging in the postoperative evaluation of acromegalic patients. Recenti Prog Med 82: 509-513.

44. Baleriaux D, Jacquemin C, Lemort M, 1990 Magnetic resonance imaging of the pituitary gland and the sella turcica region. Normal and pathological aspect. Ann Endocrinol (Paris) 51: 173-180.

45. Connor SE, Deasy NP, 2002 MRI appearances of the sphenoid sinus at the late follow-up of transsphenoidal surgery for pituitary macroadenoma. Australas Radiol 46: 33-40.

46. Dina TS, Feaster SH, Laws Jr ER, Davis DO, 1993 MR of the pituitary gland postsurgery; serial MR studies following transsphenoidal resection. AJNR Am J Neuroradiol 14: 763-769.

47. Hofmann BM, Hlavac M, Martinez R, Buchfelder M, Müller OA, Fahlbusch R, 2008 Long-term results after microsurgery for Cushing disease: experience with 426 primary operations over 35 years. J Neurosurg 108: 9-18.

48. Rees DA, Hanna FW, Davies JS, Mills RG, Vafidis J, Scanlon MF, 2002 Long-term follow-up results of transsphenoidal surgery for Cushing's disease in a single centre using strict criteria for remission. Clin Endocrinol 56: 541-251.

49. Bakiri F, Tatai S, Aouali R, et al, 1996 Treatment of Cushing's disease by transsphenoidal, pituitary microsurgery: prognosis, factors and long-term follow-up. J Endocrinol Invest 19: 572-580.

50. Barbetta L, Dall'Asta C, Tomei G, Locatelli M, Giovanelli M, Ambrosi B, 2001 Assessment of cure and recurrence after pituitary surgery for Cushing's disease. Acta Neurochir (Wien) 143: 477-482.

51. Blevins LS Jr, Christy JH, Khajavi M, Tindall GT, 1998 Outcomes of therapy for Cushing's disease due to adrenocorticotropin-secreting pituitary macroadenomas. J Clin Endocrinol Metab 83: 63-67.

52. Bochicchio D, Losa M, Buchfelder M, 1995 Factors influencing the immediate and late outcome of Cushing's disease treated by transsphenoidal surgery: a retrospective study by the European Cushing's Disease Survey Group. J Clin Endocrinol Metab 80: 3114-3120.

53. Charpentier G, de Plunkett T, Jedynak P, 1985 Surgical treatment of prolactinomas. Short- and long-term results, prognostic factors. Horm Res 22: 222-227.

54. Scanlon MF, Peters JR, Thomas JP, et al, 1985 Management of selected patients with hyperprolactinaemia by partial hypophysectomy. Br Med J (Clin Res Ed) 291: 1547-1550.

55. Maira G, Anile C, De Marinis L, Barbarino A, 1989 Prolactin-secreting adenomas: Surgical results and longterm follow-up. Neurosurgery 24: 736-743.

56. Nomikos P, Buchfelder M, Fahlbusch R, 2001 Current management of prolactinomas. J Neurooncol 54: 139-150.

57. Ciric I, Ragin A, Baumgartner C, Pierce D, 1997 Complications of transsphenoidal surgery: Results of a national survey, review of the literature, and personal experience. Neurosurgery 40: 225-236. 